\title{
СЛОЖНОСТЬ СИСТЕМЫ И ЭНТРОПИЯ
}

\section{Войтеховский Ю.Л.}

Геологический институт КНЦРАН, Anamumbl,woyt@geoksc.apatity.ru

\section{Сложность системы}

Исследуя весьма различные геологические системы (минералы и их ассоциации, горные породы и руды, геологические тела и формации), геолог закономерно - через представление об их иерархическом устройстве - приходит к необходимости количественного выражения их сложности. Сегодня для этого широко используется категория энтропии. В минералогии одним из первых её систематически применил Н.П. Юшкин, раскопавший в старых фолиантах «закон агрегации минеральных индивидов» К.Ф. Науманна [5, с. 20]

\section{три открытия энтропии}

Энтропия введена в термодинамику Р. Клаузиусом в 1865 г. как приращение теплоты в системе при данной абсолютной температуре. Её не удалось выразить в рамках статистической механики, то есть через столкновения молекул. Л. Больцман в 1872 г. предложил её неожиданную (и не принятую современниками) интерпретацию через «термодинамическую вероятность» - число микросостояний системы, отвечающих одному макросостоянию. Термодинамически закрытая система должна изменяться от менее вероятного к более вероятному состоянию с ростом энтропии. Из этих условий он вывел формулу $\mathrm{S}=-\mathrm{k} \ln \mathrm{W}$, где $\mathrm{S}$ - энтропия, $\mathrm{k}$ - постоянная Больцмана, $\mathrm{W}$ - термодинамическая вероятность. К. Шеннон $[2,7,8]$ независимо нашёл формулу $\mathrm{H}=-\Sigma \mathrm{p}_{\mathrm{i}} \log \mathrm{p}_{\mathrm{i}}$ в теории информации. А.А. Юшкевич [3] сообщает, что Э. Альфен [6] получил её в популяционной статистике ещё в 1939-1940 гг., но своевременной публикации помешала война. Н.П. Юшкин использовал энтропию в обеих интерпретациях - термодинамической [4, с. 125-135] и информационной [4, c. $168-185]$.

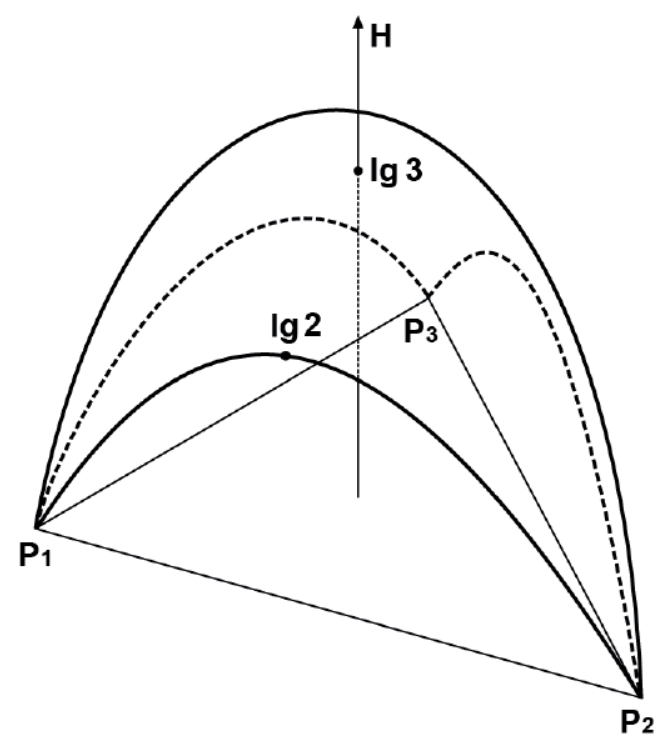

Рис. 1. График энтропии Н (в найтах, т.к. использованы десятичные логарифмы).

В геологических публикациях энтропия чаще всего используется как шкала сложности системы без осознанного принятия аксиоматики К. Шеннона или Э. Альфена. Но тогда следует хотя бы понимать особенности этой шкалы. График функции Н для двух (арки с $\mathrm{H}_{\max }=\lg 2$ ) и трёх (поверхность $\mathrm{c}_{\max }=\lg 3$ ) вероятностей показан над барицентрической диаграммой $\mathrm{p}_{1}+\mathrm{p}_{2}+\mathrm{p}_{3}=1$ на рис. 1. Легко видеть, что небольшие изменения веро-

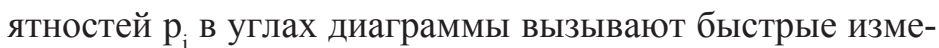
нения $\mathrm{H}$ (ножки купола крутые), а те же изменения $\mathrm{p}_{\mathrm{i}}$ в центре диаграммы не изменяют Н столь сильно (купол пологий). Таким образом, энтропия Н как шкала ведёт себя существенно по-разному в разных областях поля вероятностей. В общем случае $\mathrm{H}$ достигает максимума $\lg \mathrm{n}$ в центре поля вероятностей, когда все $\mathrm{p}_{\mathrm{i}}=1 / \mathrm{n}$, и минимума 0 в углах поля вероятностей, когда одна вероятность $\mathrm{p}_{\mathrm{i}}=1$ (остальные 0 ).

\section{Система - одна, энтропии - разные}

Из общей теории систем следует, что их сложность определяется не столько разнообразием элементов, сколько разнообразием связей. Последнее лишь потенциально ограничено разнообразием элементов и допускает вариации. Поэтому при описании сложности систем необходимо характеризовать оба аспекта. Покажем, как они связаны для 4- ... 9-вершинных выпуклых полиэдров (всего 2907), интерпретируемых как 4- ... 9-атомные кластеры [1]. 
Определим $\mathrm{H}_{\mathrm{S}}$ как шенноновскую энтропию, где $\mathrm{p}_{\mathrm{i}}$ - частоты (вероятности) вершин полиэдра (атомов кластера), взятого в комбинаторном приближении, в различных позициях его точечной группы симметрии (т.г.с.). Аналогично определим $\mathrm{H}_{\mathrm{v}}$, где $\mathrm{p}_{\mathrm{i}}$ - частоты вершин полиэдра с различными валентностями. Результаты расчётов даны на рис. 2: $\mathrm{H}_{\mathrm{S}}$ и $\mathrm{H}_{\mathrm{v}}$ не связаны жёстко с порядком группы автоморфизмов выпуклого n-вершинника. Лишь в первом приближении можно указать, что с ростом симметрии обе энтропии уменьшаются. Диапазоны их изменений и соотношения определены утверждениями 1-3.

Утверждение 1. $0 \leq \mathrm{H}_{\mathrm{S}} \leq \lg \mathrm{n}$.

Доказательство. $\mathrm{H}_{\min }=0$ в целом достигается для правильных и полуправильных $\mathrm{n}$-вершинников, а также бесконечных серий призм и антипризм (чётные $\mathrm{n} \geq 4$ ). $\mathrm{H}_{\max }=\lg \mathrm{n}$ достигается для комбинаторно асимметричных выпуклых $\mathrm{n}$-вершинников $(\mathrm{n} \geq 7)$. Для фиксированного $\mathrm{n}$ границы $\mathrm{H}_{\mathrm{S}}$ колеблются между указанными абсолютными пределами.

Утверждение 2. $0 \leq \mathrm{H}_{\mathrm{V}}<\lg \mathrm{n}$.

Доказательство. $\mathrm{H}_{\min }=0$ достигается для правильных и полуправильных n-вершинников, a также бесконечных серий призм и антипризм (чётные $\mathrm{n} \geq 4$ ). Покажем, что нет $\mathrm{n}$-вершинников, у которых все вершины имели бы разные валентности. Верно даже более сильное утверждение: у любого выпуклого n- вершинников есть по меньшей мере 4, или 3 и 2, или 3 пары вершин с одинаковыми валентностями.

Допустим, что есть выпуклый полиэдр с разноименными гранями. Рассмотрим его диаграмму Шлегеля на k-угольную грань с наибольшим числом рёбер. Построим её корону, не допуская повторов. После присоединения (k-1)-, (k-2)- ... 4- и 3-угольной граней свободными останутся 3 ребра. Таким образом, исходное допущение ошибочно. К свободным рёбрам будут присоединены 3 одноименных, 2 и 1 или 3 разноименные грани. Поскольку все возможные (3- ... k-угольные) грани уже использованы, то на полиэдре окажутся 4 , или 3 и 2 , или 3 пары одноименных граней.

Допустим, что не все k-3 типа граней представлены в короне. После того, как по одной грани каждого типа присоединены к базальной грани, свободными останутся более чем 3 ребра. Для заполнения короны придётся выбрать более чем 3 грани из меньшего, чем ранее (k-3), числа вариантов. Эти причины не могут уменьшить разнообразие граней в короне. Наконец, в силу дуальности, любой выпуклый n-вершинник имеет 4, или 3 и 2, или 3 пары вершин с одинаковыми валентностями. В общем виде оценка не может быть улучшена. Предельные случаи: тетраэдр, 3-гональная дипиримида и 6-вершинник с т.г.с. $m m 2$.

Утвержждение 3. $\mathrm{H}_{\mathrm{v}} \leq \mathrm{H}_{\mathrm{S}}$ для любого выпуклого n-вершинника, т.е. для любого $\mathrm{n}$ и т.г.с.

Доказательство. Для всех выпуклых 4- .. 9-вершинников утверждение проверено непосредственно. $\mathrm{H}_{\mathrm{v}}<\mathrm{H}_{\mathrm{S}}$ главным образом для n-вершинников с низкой симметрией, $\mathrm{H}_{\mathrm{s}}=\mathrm{H}_{\mathrm{v}}$ главным образом для n-вершинников с высокой симметрией, область перехода охватывает n-вершинники с порядками групп автоморфизмов от 2 до 12 (рис. 2). Рассмотрим произвольный n-вершинник. Вершины, эквивалентные по т.г.с., имеют равные валентности. Но различны ли валентности вершин, не эквивалентных по т.г.с.? $\mathrm{H}_{\mathrm{s}}=\mathrm{H}_{\mathrm{v}}$, если они различны, и $\mathrm{H}_{\mathrm{s}}>\mathrm{H}_{\mathrm{v}}$, если нет. Уменьшение разнообразия валентностей вершин приводит к уменьшению $\mathrm{H}_{\mathrm{v}}$ по сравнению $\mathrm{c} \mathrm{H}_{\mathrm{S}}$ согласно общим свойствам статистической энтропии $\mathrm{H}=-\Sigma \mathrm{p}_{\mathrm{i}} \log \mathrm{p}_{\mathrm{i}}$. Дадим схему доказательства.

Для любого класса выпуклых n-вершинников упорядочим последовательности чисел вершин с различными валентностями согласно алгоритму: ... p .. q ... $\left(\mathrm{H}_{1}\right) \rightarrow \ldots \mathrm{p}-1 \ldots \mathrm{q}+1 \ldots\left(\mathrm{H}_{2}\right)$, где $1 \leq$ $\mathrm{p} \leq \mathrm{q}$, и покажем, что всегда $\mathrm{H}_{1}>\mathrm{H}_{2}$. По сути, нужно сравнить по два слагаемых в разложениях $\mathrm{H}_{1}$ и $\mathrm{H}_{2}$ и доказать:

$$
-(\mathrm{p} / \mathrm{n}) \ln (\mathrm{p} / \mathrm{n})-(\mathrm{q} / \mathrm{n}) \ln (\mathrm{q} / \mathrm{n})>-[(\mathrm{p}-1) / \mathrm{n}] \ln [(\mathrm{p}-1) / \mathrm{n}]-[(\mathrm{q}+1) / \mathrm{n}] \ln [(\mathrm{q}+1) / \mathrm{n}] .
$$

Если $\mathrm{p} \rightarrow 1$, то $[(\mathrm{p}-1) / \mathrm{n}] \ln [(\mathrm{p}-1) / \mathrm{n}] \rightarrow 0$ и для $\mathrm{p}=1$ очевидно $(\mathrm{q}+1)(1+1 / \mathrm{q})^{\mathrm{q}}>1$. Для $2 \leq \mathrm{p} \leq \mathrm{q}$ следует доказать $\mathrm{p}^{\mathrm{p}} /(\mathrm{p}-1)^{\mathrm{p}-1}<(\mathrm{q}+1)^{\mathrm{q}+1} / \mathrm{q}^{\mathrm{q}}=\mathrm{f}(\mathrm{q})$. Рассмотрим $\mathrm{f}(\mathrm{q})$ как непрерывную функцию и най- 

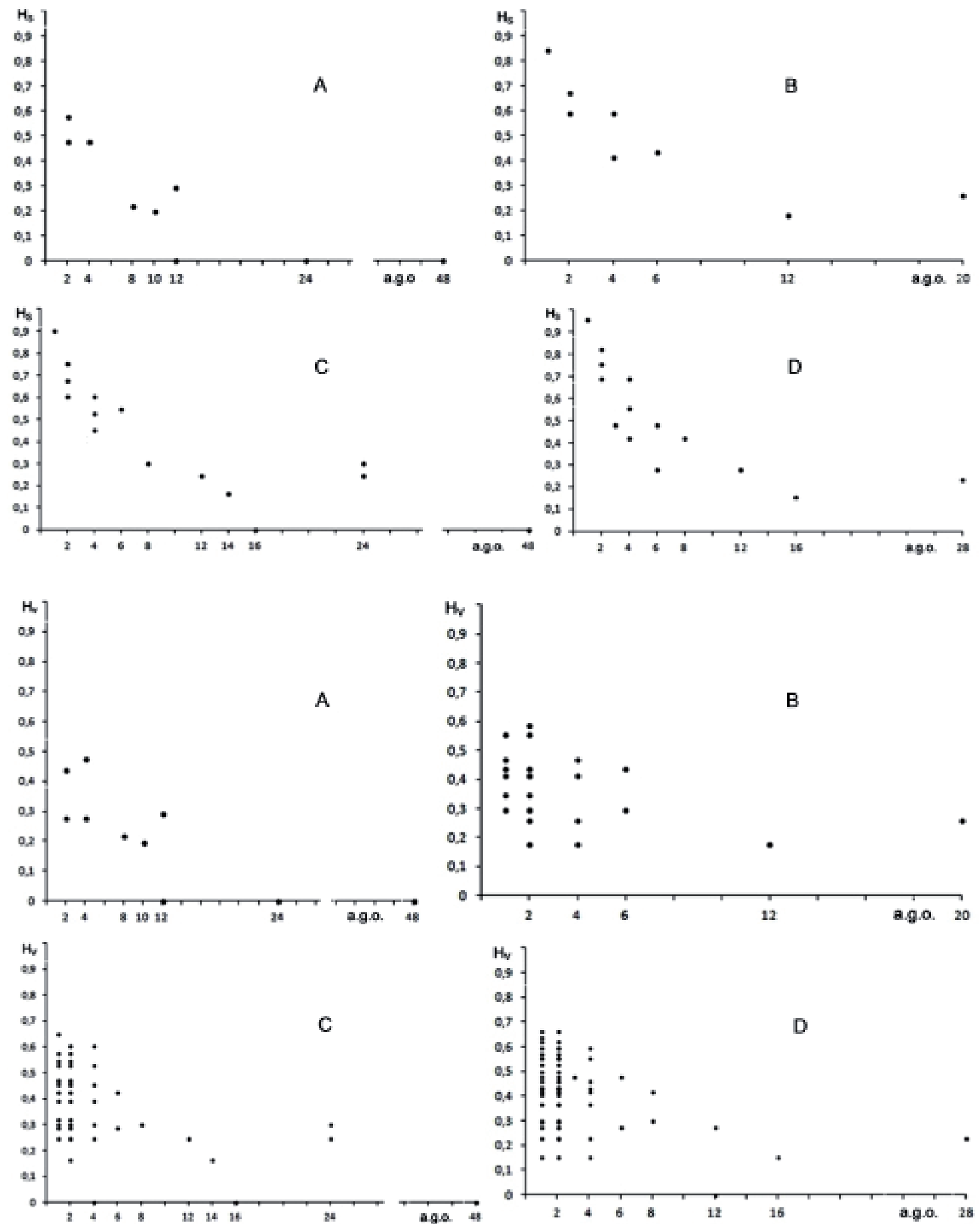

Рис. 2. Энтропии $\mathrm{H}_{\mathrm{S}}$ (вверху) и $\mathrm{H}_{\mathrm{v}}$ (внизу) для выпуклых 4- .. 6- (А, всего 10), 7- (B, 34), 8- (C, 257) и 9-вершинников $(\mathrm{D}, 2606)$ в зависимости от порядков групп автоморфизмов (a.g.o.). 
дём логарифмическую производную: $\mathrm{df} / \mathrm{dq}=\ln (1+1 / \mathrm{q}) \times(\mathrm{q}+1)^{\mathrm{q}+1} / \mathrm{q}^{\mathrm{q}}>0$. То есть, $\mathrm{f}(\mathrm{q})$ растёт с аргументом $\mathrm{q}=\mathrm{p}, \mathrm{p}+1, \mathrm{p}+2$, etc. Покажем, что неравенство верно даже для минимального аргумента $\mathrm{q}=$ $\mathrm{p}$, то есть $\mathrm{p}^{\mathrm{p}} /(\mathrm{p}-1)^{\mathrm{p}-1}<(\mathrm{p}+1)^{\mathrm{p}+1} / \mathrm{p}^{\mathrm{p}}$, в другой форме $1<(\mathrm{p}+1)^{\mathrm{p}+1}(\mathrm{p}-1)^{\mathrm{p}-1} / \mathrm{p}^{2 \mathrm{p}}=\mathrm{f}(\mathrm{p})$. Рассмотрим $\mathrm{f}(\mathrm{p})$ как непрерывную функцию и найдём логарифмическую производную: $\mathrm{df} / \mathrm{dp}=\ln \left(1-1 / \mathrm{p}^{2}\right) \times(\mathrm{p}+1)^{\mathrm{p}+1}(\mathrm{p}-1)$ $\mathrm{p}-1 / \mathrm{p}^{2 \mathrm{p}}<0$. То есть, $\mathrm{f}(\mathrm{p})$ уменьшается с ростом аргумента $\mathrm{p}=2,3,4$, etc. Действительно, $\mathrm{f}(2)=1,6875$, $\mathrm{f}(3)=1,404 \ldots, \mathrm{f}(4)=1,287 \ldots, \mathrm{f}(5)=1,223 \ldots, \mathrm{f}(6)=1,182 \ldots$ Но если $\mathrm{p} \rightarrow \infty$, то

$$
\lim f(p)=\lim (p+1)^{p+1}(p-1)^{p-1} / p^{2 p}=\lim (1+1 / p)^{p}(1-1 / p)^{p}[1+2 /(p-1)]=e \times e^{-1} \times 1=1 .
$$

Итак, $\mathrm{f}(\mathrm{p}) \rightarrow 1$ сверху, т.е. $\mathrm{f}(\mathrm{p})>1$ для любого $\mathrm{p}$. Таким образом, $\mathrm{H}_{1}>\mathrm{H}_{2}$ для любого $1 \leq \mathrm{p} \leq \mathrm{q}$ и n, что и требовалось доказать.

\section{Выводы}

Так как всякая система характеризуется разнообразием элементов и их отношений, то при описании её сложности необходимо характеризовать оба аспекта.

На примере 4- ... 9-атомных выпуклых кластеров показано, что две фундаментальные энтропии связаны между собой. Это логично, так как разнообразие отношений потенциально ограничено разнообразием элементов.

Связь симметрии и (топологической, структурной, конфигурационной) энтропии атомных систем имеет место лишь в форме весьма грубого тренда: чем выше симметрия, тем ниже энтропия.

Работа выполнена в рамках темы НИР ГИ КНЦ РАН № 0231-2015-0001.

\section{Литература}

1. Войтеховский Ю.Л., Степенщиков Д.Г. Комбинаторная кристалломорфология. Кн. 4. Выпуклые полиэдры. Т. 1. 4- .. 12-эдры. Апатиты: КНЦ РАН, 2008. 833 с. (Доступно на сайте ГИ ФИЦ КНЦ РАН).

2. Шеннон К. Работы по теории информации и кибернетике. М.: Иностр. лит., 1963. 832 с.

3. Юшкевич А.А. К истории понятий энтропии и информации: об одном предвосхищении идей К. Шеннона // Историко-математические исследования. Вып. 19. М.: Наука, 1974. С. 167-176.

4. Юшкин Н.П. Теория и методы минералогии: избранные проблемы. Л.: Наука, 1977. 291 с.

5. Юшкин Н.П. История минералогии и эволюция фундаментальных минералогических идей. Препр. 102. Сыктывкар: Коми фил. АН СССР, 1984. 52 с.

6. Halphen E. L'analyse intrinsèque des distributions de probabilité // Publ. Inst. Stat. Uni. Paris. 1957. V. 6. N 2. P. 77-159.

7. Shannon C.E. The mathematical theory of communication // Bell Syst. Tech. J. 1948. V. 27. P. 379-423, 623-656.

8. Shannon C.E., Weaver W. The Mathematical Theory of Communication. Urbana: Uni. Illinois Press, 1949. 117 p. 